\title{
Vezatin, a ubiquitous protein of adherens cell-cell junctions, is exclusively expressed in germ cells in mouse testis
}

\author{
Vincent Hyenne ${ }^{1}$, Juergen C Harf ${ }^{2}$, Martin Latz ${ }^{2}$, Bernard Maro ${ }^{1,3}$, Uwe Wolfrum ${ }^{2}$ \\ and Marie-Christine Simmler ${ }^{1}$ \\ ${ }^{1}$ Biologie Cellulaire du Développement, UMR 7622, CNRS, Université Pierre et Marie Curie, 9 Quai St Bernard, \\ 75252 Paris cedex 05, France, ${ }^{2}$ Institut für Zoologie, Johannes Gutenberg-Universität, Müllerweg 6, 55099 Mainz, \\ Germany and ${ }^{3}$ Sackler Faculty of Medicine, Tel Aviv University, Ramat Aviv 69978, Israel \\ Correspondence should be addressed to M-C Simmler and B Maro; Email: msimmler@ccr.jussieu.fr; maro@ccr.jussieu.fr
}

U Wolfrum and M-C Simmler contributed equally to this work

$\checkmark$ Hyenne is now at Université de Montréal, Institut de Recherche en immunologie et Cancérologie, 2900 boulevard ÉdouardMontpetit, Pavillon Marcelle-Coutu, Montréal, Quebec, Canada H3T 1J4

M-C Simmler is now at Trafic Membranaire et Morphogenèse Neuronale et Epithéliale, UMR 7592, CNRS, Institut Jacques Monod, Universités Pierre et Marie Curie et René Descartes, Tour 43-44, 2ème étage, 2 Place Jussieu, 75251 Paris cedex 05, France

\begin{abstract}
In the male reproductive organs of mammals, the formation of spermatozoa takes place during two successive phases: differentiation (in the testis) and maturation (in the epididymis). The first phase, spermiogenesis, relies on a unique adherens junction, the apical ectoplasmic specialization linking the epithelial Sertoli cells to immature differentiating spermatids. Vezatin is a transmembrane protein associated with adherens junctions and the actin cytoskeleton in most epithelial cells. We report here the expression profile of vezatin during spermatogenesis. Vezatin is exclusively expressed in haploid germ cells.

Immunocytochemical and ultrastructural analyses showed that vezatin intimately coincides, temporally and spatially, with acrosome formation. While vezatin is a transmembrane protein associated with adherens junctions in many epithelial cells, it is not seen at the ectoplasmic specializations, neither at the basal nor at the apical sites, in the seminiferous epithelium. In particular, vezatin does not colocalize with espin and myosin VIla, two molecular markers of the ectoplasmic specialization. In differentiating spermatids, ultrastructural data indicate that vezatin localizes in the acrosome. In epididymal sperm, vezatin localizes also to the outer acrosomal membrane. Considering its developmental and molecular characteristics, vezatin may be involved in the assembly/stability of this spermatic membrane.

Reproduction (2007) 133 563-574
\end{abstract}

\section{Introduction}

Mammalian male germ cells progressively differentiate in the testis in a complex morphogenetic process and mature into functional sperm in the epididymis. Within the testis, differentiating germ cells migrate through the seminiferous epithelium, from the basal lamina to the lumen, where spermatozoa are released. During this phase, round haploid spermatids change into elongated and highly polarized spermatozoa: the chromatin condenses, the nucleus elongates, the acrosome elaborates over the nucleus and the tail, containing mitochondria, forms in its proximal region. Once the spermatozoon is released to the lumen, it travels through rete testis and epididymis. There, it is confronted to a complex and specific microenvironment, which varies along the epididymis (Johnston et al. 2005, Sullivan et al. 2005). This complex fluid interacts with the sperm surface and induces its acquisition of motility and fertilizing abilities (Orgebin-Crist 1967, Zhou et al. 2004). The maturation phase is accompanied by numerous dynamic changes in the localization and the processing of proteins and glycolipids, both at the sperm surface and within the acrosome (Yoshinaga \& Toshimori 2003, Burkin et al. 2004, Christova et al. 2004). However, the molecular basis for sperm maturation remains largely unknown.

Close interactions between somatic Sertoli cells and differentiating germ cells - through a unique and highly dynamic actin-based adhesion complex named the ectoplasmic specialization - are essential for a proper spermiogenesis. Two types of ectoplasmic specializations 
are present in the testis: 'the basal ectoplasmic specialization' between adjacent Sertoli cells, contributing to the blood testis barrier, and 'the apical ectoplasmic specialization' between Sertoli cells and elongating spermatids. The apical ectoplasmic specialization is thought to control adhesion and release of the spermatid, as well as its precise orientation and positioning, and the correct shaping of its head (Mruk \& Cheng 2004). Three actin-based adhesion complexes are located at the apical ectoplasmic specialization: the integrin-laminin, the nectin-afadin, and the cadherin-catenin complex (Cheng \& Mruk 2002). Expression of the integrin-laminin complex, usually implicated in cell-extracellular matrix interactions, coincides with assembly and disassembly of apical ectoplasmic specialization in the testis (Palombi et al. 1992, Salanova et al. 1995, Mulholland et al. 2001). This unique implication in cell-cell interactions may be related to the highly dynamic properties of the apical ectoplasmic specialization. The nectin-afadin complex links spermatid to the Sertoli cell through a heterotypic interaction between nectin-2, located exclusively on the Sertoli side of the apical ectoplasmic specialization and nectin-3, located on the spermatid side (Ozaki-Kuroda et al. 2002). Pvrl-2 (previously known as Nectin-2) knockout induces abnormal development of the spermatid head resulting in misshaped nuclei, presence of mitochondria in the head, and a disorganized middle piece (Bouchard et al. 2000). Pvrl-3 (previously known as Nectin-3) knockout also induces sterility in the mouse (Inagaki et al. 2005). JAMC (junctional adhesion molecule C; now called JAM3), a transmembrane protein of the immunoglobulin superfamily has been shown to localize to the apical ectoplasmic specialization in the testis. Jam3 knockout completely abrogates polarization of the spermatid head by disturbing recruitment of the PAR6-Cdc42-PKC cell polarity complex (Gliki et al. 2004). More recently, Igsf4 (Ra175/Tslc1/Nectin-like 2/SynCAM) was described as a novel cell adhesion protein of the immunoglobulin family strongly expressed at the cell junctions between Sertoli cells and elongating/elongated spermatids. The absence of Igsf4 in Igsf4 ${ }^{-1-}$ testes results in the absence of mature elongated spermatid and leads to a phenotype of oligoastheno-teratozoospermia (Fujita et al. 2006). Pvrl-2, Pvrl3, Jam3, and Igsf4 knockout illustrate the importance of the interactions between spermatids and Sertoli cells during spermiogenesis. Despite its well-established and documented function in various epithelia, the role of the cadherin-catenin complex in the testis still remains elusive. The three basic members of the complex, $\mathrm{N}$-cadherin, $\alpha_{-}$, and $\beta$-catenin are present at the ectoplasmic specialization (Newton et al. 1993, Byers et al. 1994, Goossens \& van Roy 2005). However, the only functional evidence for a direct implication of the complex in spermiogenesis comes from in vitro data: application of an anti-N-cadherin antibody on cultured Sertoli cells, abrogates Sertoli-germ cell interactions (Newton et al. 1993).
In various epithelial cells, vezatin, a second transmembrane protein of adherens cell-cell junctions based on the $\mathrm{Ca}^{2+}$-dependent cadherin family, anchors unconventional myosin VIla to the cadherin-catenin complex (Kussel-Andermann et al. 2000, Hyenne et al. 2005). At the site of adherens junctions, vezatin could modulate cell-cell adhesion by creating a tension between the plasma membrane and the actin cytoskeleton. Vezatin is implicated in the regulation of the cadherin-catenin complex by acting at cell-cell contacts and in the nucleus (Hyenne et al. 2005). In mouse early embryo, vezatin may regulate E-cadherin expression (Hyenne et al. 2005).

In this study, we show that vezatin is expressed in the testis, but not, as expected, at ectoplasmic specialization in Sertoli cells. On the contrary, vezatin is only present in the acrosome of the haploid germ cells as evidenced by immunocytochemistry analyses applying light and electron microscopy. In the mature sperm, vezatin seems to be both in the acrosome and at the outer acrosomal membrane.

\section{Materials and Methods RT-PCR}

For RT-PCR experiments, seminiferous tubules were dissected out and mRNA was extracted (QIAshredder and RNeasy kit, Qiagen). A aliquot of 100 ng mRNA was submitted to reverse transcription (Superscript, Invitrogen). Each reaction was carried out in parallel without reverse transcriptase to control for false-positive PCR amplifications. PCR amplification was performed with the TaKaRa LA Taq polymerase (Takara Bio Inc). Samples were denatured at $94{ }^{\circ} \mathrm{C}, 2 \mathrm{~min}$ and then subjected to 35 cycles of amplification $\left(94{ }^{\circ} \mathrm{C}, 15 \mathrm{~s} ; 62{ }^{\circ} \mathrm{C}, 20 \mathrm{~s} ; 68^{\circ} \mathrm{C}\right.$, $4 \mathrm{~min}$ ). $5^{\prime}$-End primers used in our experiments were the following: Veza5, AGGATGACACCGGAGTTTGACGAAG, and three specific $3^{\prime}$-end primers containing the stop codon: Veza3-2.4, TTATTTTTCCTCCACCTCATTTTC; Veza3-1.9, CTAATT-CCACTCGGCCTTTACC; Veza3-0.8, TCATGTTAAGTCCATGATATGGGTAAAAG. Expected amplicons sizes were 2343 bp for vezatin 2.4 isoform, $1854 \mathrm{bp}$ for vezatin 1.9 isoform, and $831 \mathrm{bp}$ for vezatin 0.8 isoform. Three minor spliced isoforms were identified on agarose gels only with vezatin 1.9 isoform. The three potential products were confirmed using $5^{\prime}$-end primers overlapping two exons: exon5/7F, GGGGATTTACTCTCTACССССТGAACTCTG, exon6/8F, GGGATTTACTCT-GGTCAGTGCTGCTTGCTC and exon5/8F, GGGGATTTACTCTGTTTTGTTCCAGCTCTG and $3^{\prime}$ end primer in exon 8: Ve8R, ATGGAGCTGTAAGCTGCGCAC-TGCTGTGTG. Expected amplicons size of 486 (exon 6 skipping), 462 bp (exon 7 skipping), and $319 \mathrm{bp}$ (exon $6+7$ skipping) were obtained corresponding to the three deletion events. 


\section{Antibodies}

The rabbit anti-vezatin antibodies, affinity-purified antivezatin, and immune serum anti-hA34P1 (1:200 for immunofluorescence, 1:1000 for western blot) and the monoclonal anti-myosin VIla (1:300 for immunofluorescence) have been previously characterized (KusselAndermann et al. 2000, Hyenne et al. 2005). A biotinylated-vezatin fusion protein (Hyenne et al. 2005) was used for preadsorption of the anti-vezatin antibody as control experiments and preimmune serum was used as a control for the anti-hA34P1. The monoclonal anti-espin (1:80 in immunofluorescence) was purchased from BD Bioscience PharMingen. The phalloidin-tetramethylrhodamine conjugate was purchased from Sigma (1:1000). The fluorescein-conjugated and rhodaminconjugated anti-rabbit or anti-mouse IgG antibodies were purchased from either KPL company, Niles or from Invitrogen (Alexa 488- or 568-conjugated, Molecular probes, Invitrogen). Lectin peanut agglutinin (PNA from Arachis hypogaea) Alexa 568-labeled, wheat germ agglutinin (WGA from Triticum vulgare) tetramethylrhodamine conjugate and MitoTracker Red 589 Dye were purchased from Molecular probes (Invitrogen) and used at $2,5 \mu \mathrm{g} / \mathrm{ml}$, and $2 \mathrm{nM}$ respectively.

\section{Immunofluorescence}

SWISS:RJ, C57BL/6N (NIH), and 129S2/SvPas mice were purchased from Elevage Janvier (Le Genest-St-Isle, France). Dissected testes or epididymides from adult SWISS:RJ or $(\mathrm{C} 57 \mathrm{BL} / 6 \mathrm{~N} \times 129 \mathrm{~S} 2) \mathrm{F} 1$ were fixed in $4 \%$ paraformaldehyde in $0.1 \mathrm{M}$ phosphate buffer, $\mathrm{pH} 7.3$ (paraformaldehyde/PBS) solution for $1 \mathrm{~h}$ at $4{ }^{\circ} \mathrm{C}$, immerged in sucrose 20\%/PBS for $24 \mathrm{~h}$ and embedded in Tissue-Tek (Sakura Finetek, Europe BV, Leiden, The Netherlands). In cold methanol, $10 \mu \mathrm{m}$ cryosections were postfixed for $3 \mathrm{~min}$ (or in 4\% PFA/PBS solution for $10 \mathrm{~min}$ in case of F-actin labeling using the phalloidin stain), quenched with $\mathrm{NH}_{4} \mathrm{Cl}$ for 15 min, incubated in $20 \%$ normal goat serum for $1 \mathrm{~h}$ and labeled with primary rabbit anti-vezatin antibodies followed by secondary anti-rabbit Alexa 488-conjugated IgG for $1 \mathrm{~h}$ at room temperature. Colabeling with primary mouse anti-espin (or mouse anti-myosin VIla) antibodies for $1 \mathrm{~h}$ at room temperature, was followed by an incubation with secondary Alexa 488-conjugated anti-rabbit and Alexa 568-conjugated anti-mouse $\lg \mathrm{G}$ for $1 \mathrm{~h}$ at room temperature. Colabeling of anti-vezatin antibodies with lectins consisted of a first incubation with primary rabbit anti-vezatin antibdodies alone for $1 \mathrm{~h}$ at room temperature, followed by an incubation with Alexa 568- (or tetramethyl-rhodamine-) conjugated lectins for $1 \mathrm{~h}$ at room temperature together with the secondary antirabbit Alexa 488-conjugated IgG. The phalloidintetramethyl-rhodamine conjugate was used for $10 \mathrm{~min}$ after secondary Alexa 488-conjugated IgG. DNA was labeled for 5 min with Hoechst stain solution (Sigma). All reagents were diluted in $\mathrm{PBS} / 1 \%$ bovine serum albumin. To preserve fluorescent signals, we used FluorSave Reagent mounting solution (Calbiochem, Merck KGaA, Darmstadt, Germany). Mature spermatozoa were flushed from epididymis on a glass surface in PBS and fixed for 15 min at room temperature with freshly prepared 3\% PFA/PBS, $0.1 \mathrm{M}$ cacodylate buffer $\mathrm{pH} 7.3,1 \mathrm{mM} \mathrm{CaCl}_{2}$, and $\mathrm{MgCl}_{2}$.

\section{Western blot}

Tissue samples from adult SWISS:RJ mice were homogenized in RIPA extraction buffer $(50 \mathrm{mM}$ Tris- $\mathrm{HCl}$ pH 7.4, $150 \mathrm{mM} \mathrm{NaCl}, 0.1 \%$ SDS, $2 \mathrm{mM}$ EDTA, 1\% NP-40, 0.5\% sodium deoxycholate, $1 \mathrm{mM}$ sodium vanadate, $30 \mathrm{mM}$ sodium pyrophosphate) by ultrasonic vibration (Labsonic 1510, Braun Melsungen AG, Melsungen, Germany). A protein inhibitor mixture (leupeptin $0.42 \mu \mathrm{g} / \mathrm{ml}$, pepstatin $0.83 \mu \mathrm{g} / \mathrm{ml}$, and aprotinin $0.83 \mu \mathrm{g} / \mathrm{ml}$; Sigma-Aldrich) was added. The concentration of proteins in the homogenate was established by the BCA(bicinchoninic acid)-method. A measure of $10 \mu \mathrm{g}$ proteins were added to each lane of $10 \%$ gels (SDS-PAGE). Proteins were blotted on to a PVDF membrane in a tank blotter (Genie blotter, Idea Scientific Company, Minneapolis, USA). The membranes were blocked by the Applichem blocking reagent (Applichem $\mathrm{GmbH}$, Darmstadt, Germany). Monoclonal anti-actin antibodies (clone C4) were kindly provided by J L Lessard, Cincinnati, Ohio, USA (Lessard 1988) and were used in a 1:2000 dilution. Secondary antibodies (goat anti-rabbit IR dye 680 and goat anti-mouse IR dye 800) were purchased from Molecular Probes (Invitrogen). Detection and relational quantification was carried out by the infrared imaging system Odyssey of LI-COR Biosciences (Lincoln, $N E$, USA), using $\beta$-actin as standard.

\section{Electron microscopy}

For conventional electron microscopy, freshly isolated mouse testes were conventionally fixed, according to Wolfrum (1992). Specimens were fixed in a mixture of $2.5 \%$ glutaraldehyde and $1 \%$ paraformaldehyde in $0.1 \mathrm{M}$ cacodylate buffer $(\mathrm{pH} 7.2)$, and postfixed in buffered $1 \%$ osmium tetroxide in cacodylate buffer. Subsequently, the washed specimens were dehydrated to $100 \%$ ethanol $/ 100 \%$ propylene oxide and embedded in araldite resin, followed by polymerization at $60^{\circ} \mathrm{C}$. For immunoelectron microscopy, isolated testes of C57BL/6] mice were fixed and embedded in $L R$ White resin (Electron Microscopy Sciences, Fort Washington, PA, USA; Wolfrum \& Salisbury 1998). In short, we fixed the testes in $0.1 \%$ glutaraldehyde and $4 \%$ paraformaldehyde in $0.1 \mathrm{M}$ phosphate buffer $(\mathrm{pH} 7.4)$ for $3 \mathrm{~h}$ at room temperature. During fixation, the testes were dissected into small pieces. Fixed specimens were washed in 
phosphate buffer, dehydrated to $98 \%$ ethanol, embedded in L R White, and polymerized at $4{ }^{\circ} \mathrm{C}$ under indirect u.v. light for at least $48 \mathrm{~h}$. Sperm specimens for immunoelectron microscopic analyses were isolated and fixed in $0.1 \%$ glutaraldehyde and $4 \%$ paraformaldehyde in $0.1 \mathrm{M}$ phosphate buffer $(\mathrm{pH} 7.4)$. Isolated sperms were obtained either from dissected epididymes (C57BL/6 mice) or from ejaculates (human, pig, and bovine). The specimens were embedded in $L$ R White resin as described above. Latter specimens were kindly provided

A

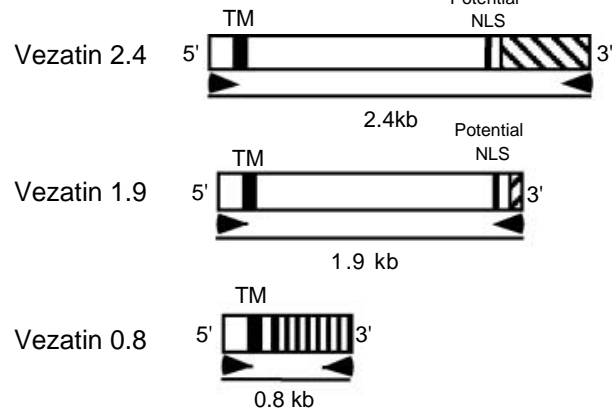

B

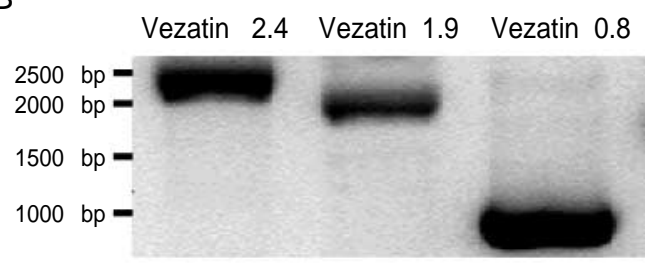

C

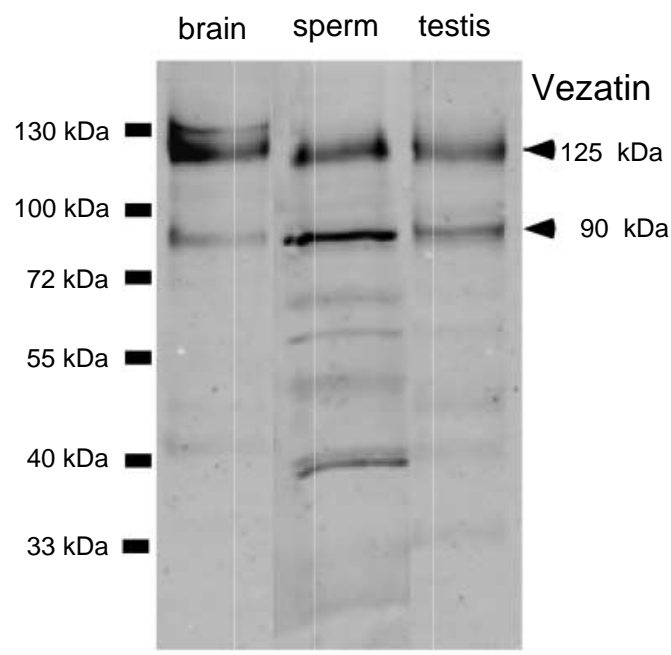

D

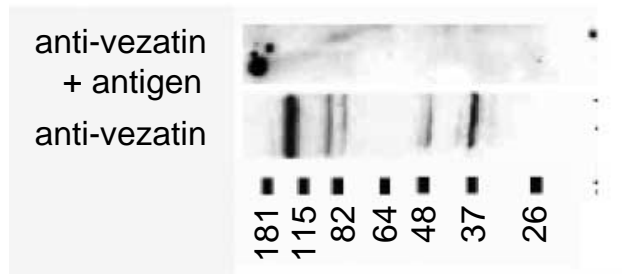

by Dr Günther Kamp (Institut für Zoologie, Johannes Gutenberg-Universität, Germany). Ultrathin sections collected on Formvar-coated nickel grids were etched with saturated sodium periodate (Sigma-Aldrich) before immunogold labeling. They were incubated with primary antibodies for $60 \mathrm{~h}$ in blocking solution followed by an incubation for $2 \mathrm{~h}$ with the secondary antibodies, $10 \mathrm{~nm}$ colloidal gold coupled anti-rabbit Fab fragment from goat (Aurion, Wageningen, The Netherlands) or anti-rabbit $1.4 \mathrm{~nm}$ Nanogold conjugated to Fab fragment from goat (Nanoprobes, Yaphank, NY, USA). The immunogold label with Nanogold was silverenhanced according to Danscher (1981). For controls, vezatin antibodies preadsorbed with the antigen before immunolabeling and the primary antibody was omitted. No immunolabeling above background was observed on control sections. Counterstained ultrathin sections were analyzed by electron microscope FEI Tecnai 12 Biotwin (Eindhoven, The Netherlands). Images were processed with Adobe Photoshop (Adobe Systems Incorporated San Jose, CA, USA).

\section{Animal care and use statement}

All animals used in experiments reported in this publication were handled by persons qualified by institutional committee according to ethical welfare guidelines of CNRS and German authorizing agencies.

\section{Results}

\section{Vezatin is expressed in mouse testis}

We analyzed vezatin mRNA expression in the testis by RT-PCR using primers specific to all mouse vezatin isoforms available in databases. Applying these isoform-specific

Figure 1 Vezatin transcript and protein expression in mouse testis. (A) Schematic representation of the three major isoforms of vezatin, corresponding to the gene sequences AY753561 (vezatin-2.4), AY753562 (vezatin-1.9; Hyenne et al. 2005), and DQ025533 (vezatin 0.8 ; Hyenne et al. unpublished observations) respectively. The last exon specific to each isoform is labeled with various motifs. Arrows indicate the localization of PCR primers used to amplify the testis mRNA. TM, transmembrane domain; potential NLS, Bipartite Nuclear Localization Signal. (B) RT-PCR analysis of mRNA of wild-type testis tissue. (C) Analysis of vezatin expression by western blot on wild-type brain, pure sperm, and testis extracts, using immunopurified anti-vezatin antibody. Two major specific bands at 90 and $125 \mathrm{kDa}$ (arrows) and one band at $40 \mathrm{kDa}$ inconstantly detected, are observed in western blot with the anti-vezatin antibodies using testis extracts. (D) Anti-vezatin antibodies were preadsorbed for $2 \mathrm{~h}$ with a vezatin-biotinylated immunogen (aa 364-617 of the sequence AY753562, vezatin 1.9, (Hyenne et al. 2005) fixed on a streptavidin-coated resin, prior to incubation with the membrane loaded with extracts from whole male reproductive tissue (testis and epididymis tissues). The two major testis-specific bands at 90 and $125 \mathrm{kDa}$ and the one migrating at $40 \mathrm{kDa}$, most probably corresponding to both testis and epididymal sperm proteins, are totally lost after the competition with the immunogen. 
primers, we detected three major alternatively spliced isoforms, vezatin 2.4 , vezatin 1.9, and vezatin 0.8 (Fig. 1A and $B$ ). All of them had the same $5^{\prime}$-end and include exon 5 that encodes the transmembrane domain (Fig. 1A). They diverged by exons encoding their intracytoplasmic region. vezatin 2.4 (780 aa, estimated molecular mass, $86 \mathrm{kDa}$; AY753561) only differed from vezatin 1.9 by its last exon (617 aa, estimated molecular mass, 68 kDa; AY753562). Both carried a predicted coiled-coil domain and nuclear localization sequence in their cytoplasmic regions and were shown to be expressed in mouse preimplantation embryo (Hyenne et al. 2005). vezatin 0.8 only shared the first five exons with vezatin 1.9 and vezatin 2.4 (Hyenne et al. unpublished observations). It had a shorter intracytoplasmic region with no known domain described yet. It corresponded to a protein of 276 aa with an estimated molecular mass of $31 \mathrm{KDa}$ (Fig. 1A). We also detected three alternatively spliced variants of the vezatin 1.9 isoform, expressed in very minor quantities. All the three were detected only by nested PCR. They potentially corresponded to splicing of exon $6(-138 \mathrm{nt}), 7(-147 \mathrm{nt})$ or 6 together with 7 ( $-285 \mathrm{nt}$ ) (see Materials and Methods). Only splicing of exon 6 respected the open reading frame encoding a putative protein of an expected molecular mass of $63 \mathrm{kDa}$ (data not shown).

We examined vezatin protein expression in the whole testis by western blot. The immunoblots revealed that two major isoforms of vezatin were expressed, migrating at apparent molecular mass of 90 and $125 \mathrm{kDa}$. Those isoforms may correspond to the translation of either one or the two longest mRNA expressed in the testis, vezatin 2.4 and vezatin 1.9 , if we assumed that the difference between the observed and the predicted molecular mass was due to posttranslational modifications. Several other minor bands were also observed. They may be due to non-specific reaction of the anti-vezatin antibody, to cleavage products of the main isoforms, or alternatively to spliced variants of the main isoforms. We also analyzed vezatin protein expression in extracts from sperm flushed from the epididymis. In these isolated sperm extracts, we also observed both major bands besides several minor products (Fig. 1C). In Fig. 1D, the specificity of the polyclonal affinity-purified anti-vezatin antibody is demonstrated. In the present western blot experiments, the immunoreactive bands (see Fig. 1C) were completely abolished after preadsorption of the antibodies with a vezatin-biotinylated immunogen fixed on a streptavidin-coated resin, prior to incubation of the membrane (Hyenne et al. 2005).

\section{Vezatin localizes exclusively in the acrosome of the germ cells}

To determine cellular localization of the vezatin, we analyzed testis sections and isolated sperms by immunofluorescence using two different anti-vezatin antibodies (see Materials and Methods). The two antibodies gave the same results. As shown in Fig. $2 \mathrm{~A}$ and $\mathrm{B}$, vezatin was present in the adluminal but not in the basal part of the seminiferous tubules (see also a single confocal section in Fig. 3D). No labeling was noted using the preadsorbed immunoserum (Fig. 2C) or the preimmune IgG (Fig. 2D).

We observed distinct vezatin patterns depending on the tubule differentiation stage. To follow vezatin localization during spermiogenesis, we used fluorescent-labeled lectins, PNA (peanut agglutinin from Arachis hypogaea), and WGA (wheat germ agglutinin from Triticum vulgare) as molecular markers of the developing acrosome. While PNA, specific to $\beta$-galactosyl residues, binds preferentially to the plasma membrane/outer acrosomal membrane, WGA, specific to $\mathrm{N}$-acetylglucosamine and/or sialic acid, localizes preferentially to the equatorial segment and posterior head of sperm (Benoff 1997). Sixteen steps of mouse spermiogenesis were described based on the formation of the acrosome and the flagellum, as well as on the nuclear morphology and the mitochondrial location. Round spermatids are observed in steps 1-8 (S1-S8) and elongating/elongated/condensed spermatids are observed in steps 9-16 (S9-S16; Russell et al. 1990). These steps can be visualized by the labeling of any of the two fluorescent lectins, since they almost overlap at all stages of acrosome development. As shown in Figs 2 (conventional microscopy) and 3 (confocal microscopy), vezatin staining followed acrosome biogenesis (Fig. 2E-I and see single confocal sections in Fig. 3A). First, vezatin labeling adopted a rounded shape that encircled an unstained center, the acrosome vesicle (or acrosomal granule), around steps S2-S3 (Fig. 2E). Then, the antivezatin stained area enlarged over the elongating nucleus following the acrosome flattening (Fig. 2F) and finally, adopted a hooked shape over the acrosome of the elongated spermatid (Fig. 2G and $\mathrm{H}$ ). In the mature epididymal sperm, anti-vezatin immunofluorescence was present at the very tip and at the dorsal part of the sperm head (Fig. 2J, schematized in Fig. $2 \mathrm{l}$ and see a single confocal section of a wild type epididymis in Fig. 3B). The cytoplasmic droplet stained by both fluorescent lectins was commonly located at the distal end of the midpiece in a mature sperm of the middle caput (see the staining for the PNA lectin, Fig. 2J). A weak anti-vezatin staining was also observed in the tail of the spermatozoa. Mitochondria sheath-labeled with Mitotracker dye was normally labeled (data not shown).

To determine whether vezatin is present also at ectoplasmic specializations, the testis-specific adherens junctions, we looked at different markers (espin, myosin VIla and F-actin) that are all enriched in these junctions (Bartles et al. 1996, Wolfrum et al. 1998). We did not observe a colocalization of vezatin with espin (Fig. 4A and $\mathrm{B}$, see also single confocal sections in Fig. 3C left panel), myosin VIla (Fig. 4C, see also single confocal sections in Fig. 3C right panel), and F-actin (Fig. 4D). As seen in Fig. 4A-C, myosin VIla and espin also localize to 


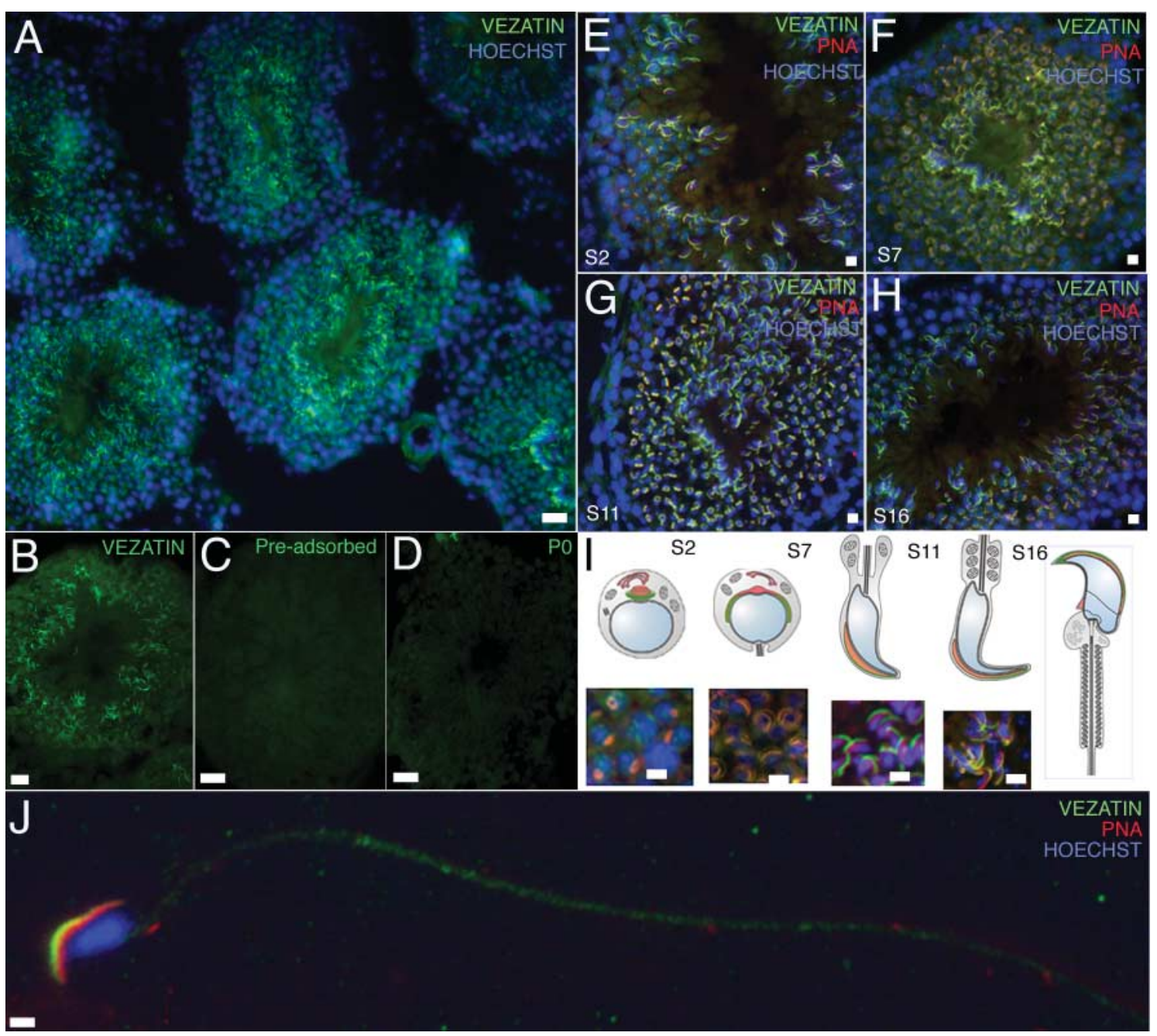

Figure 2 Indirect immunofluorescence localization of vezatin in wild-type testis cryosections and epididymal flushed sperm, observed with a conventional immunofluorescence microscope. Specific cell associations from step S1-S16 that defined the stages of the cycle of the seminiferous epithelium (stages 1-12) are present within successive overlapping reiterated regions, along the length of a seminiferous tubule (Kotaja et al. 2004). (A) Wild-type tubules stained for vezatin (green) and DNA (blue) and (B) for vezatin only using immunopurified anti-m34, (C) preadsorbed anti-m34 or (D) preimmune hA34P1 serum. (E-H) Wild-type tubules are stained for vezatin (green), nuclei (blue), and PNA (red) during the biogenesis of the acrosome, (E) step S2 in Golgi phase, (F) step S7 in cap phase, (G) step S11 in elongation/acrosomal phase and (H) step S16 in maturation phase. (I) Schematized representation of vezatin staining and images at higher magnification corresponding to steps S2, S7, S11, and S16. (J) Wild-type sperm flushed from the epididymis stained for vezatin (green), PNA (red), and DNA (blue). Last image in (I) represents a schematic view of a spermatozoa in the epididymis. Bars: (A-D) $30 \mu \mathrm{m}$, (E-H) $5 \mu \mathrm{m}$, and (I) $1 \mu \mathrm{m}$.

the basal region of the seminiferous epithelium, most probably to the subplasmalemmal web underneath the cell membrane on the basal lamina as already described, at least for myosin VIIa, in the intestinal epithelium (Drenckhahn \& Groschel-Stewart 1980).

\section{Vezatin partially localizes to the outer acrosomal membrane, in epididymal sperm}

To confirm and more precisely define these immunofluorescence results, we followed vezatin localization by immunoelectron microscopy in spermatids and epididymal sperm. In anti-vezatin-labeled ultrathin sections through spermatids at the Golgi phase, only a few colloidal gold particles were present in the acrosome (Fig. 5A). In the cap phase, anti-vezatin immunogold labeling was clearly present in the nascent acrosome of round spermatids (Fig. 5B). Later, in elongated spermatids at the elongation and maturation phases (Fig. 5C and D), vezatin was still localized to the acrosome and was excluded from the cytoplasm of Sertoli cells. Furthermore, no vezatin staining was observed at the plasma membrane of the spermatid, where the germ cell part of the apical ectoplasmic specialization is located. Immunoelectron microscopy on ultrathin sections through mature mouse spermatozoon isolated from the epididymis revealed that anti-vezatin immunolabeling was not only detectable in the acrosome as in the testis 

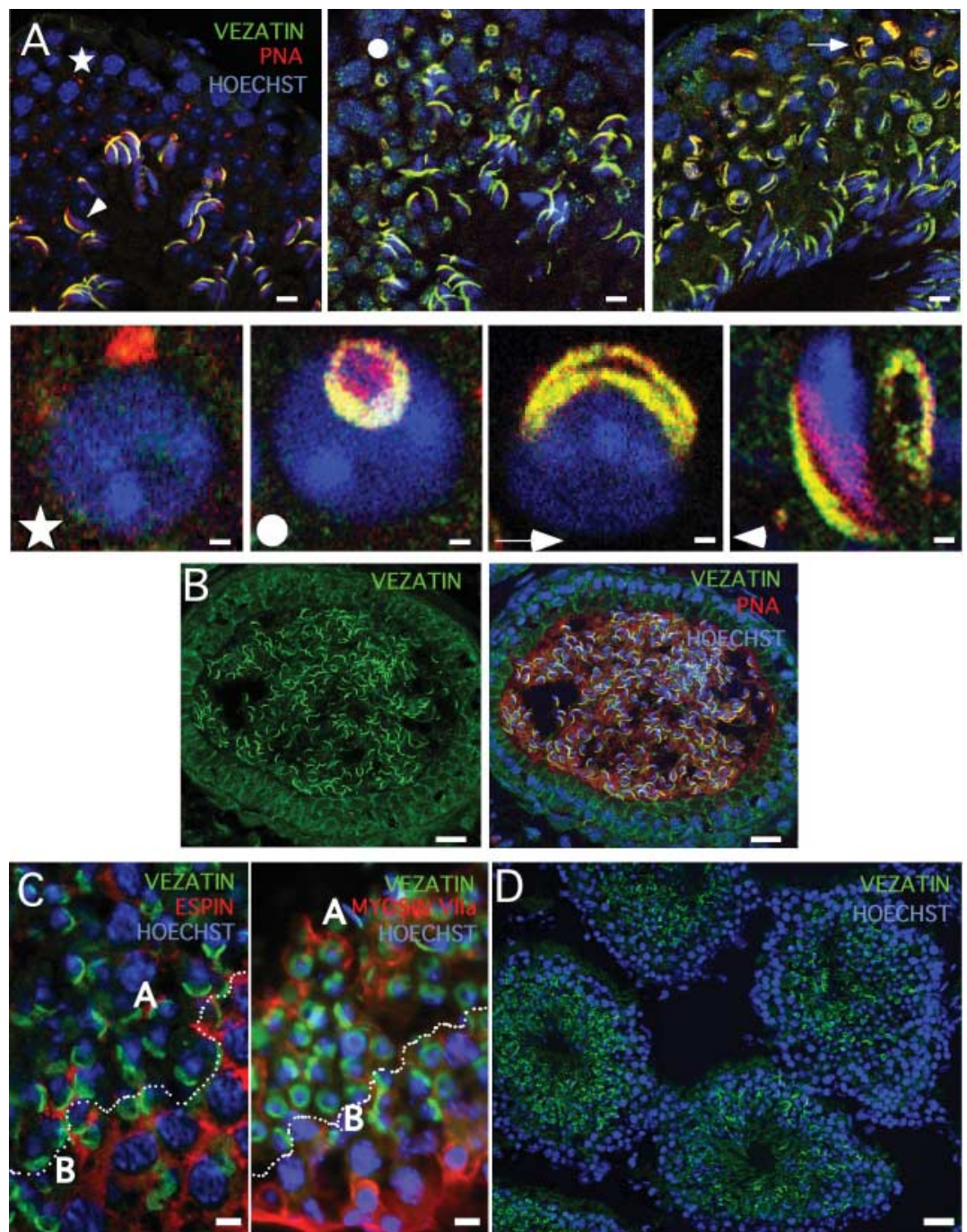

Figure 3 Single confocal sections. (A) Wild-type tubules stained for vezatin (green), nuclei (blue), and PNA (red). Star, dot, arrow, and arrowhead indicate spermatids at high magnification at steps S1, S3, S7, and S11 of spermiogenesis. (B) Wild-type epididymal sections stained for vezatin alone (green) or vezatin (green), PNA (red), and DNA (blue). Interestingly, we also observed vezatin at the apical membrane of epithelial cells in the initial segment of wild-type epididymes. $\beta$-catenin, another member of adherens junctions, too localizes to the apical membrane in those cells (DeBellefeuille et al. 2003). (C) A wild-type tubule stained for vezatin (green), nuclei (blue), and espin (left side, red) or myosin VIla (right side, red). $\mathrm{A}$, this indicates the region harboring apical ectoplasmic specializations; $\mathrm{B}$, this indicates the region harboring the basal ectoplasmic specializations. A dashed line indicates the border of basal ectoplasmic specializations. (D) Wild-type tubules stained for vezatin (green) and DNA (blue). Bars: (A) $5 \mu \mathrm{m}$ and $500 \mathrm{~nm}$ at higher magnification respectively, (B) $20 \mu \mathrm{m}$, (C) $5 \mu \mathrm{m}$, and (D) $10 \mu \mathrm{m}$.

tissue but was also partially present at the sperm outer acrosomal membrane (Fig. 6A). Analyses of the isolated sperms of other mammals showed an identical antivezatin labeling (Fig. 6B-D) suggesting that vezatin might localize both in the acrosome and at the outer acrosomal membrane in epididymal spermatozoa. Thus, this appears to be a common phenomenon in mammals.

\section{Discussion}

Vezatin is a common protein associated with adherens junction in most epithelial cells. Our present data obtained by both light and electron microscopy, and by using two different antibodies show that the expression of vezatin in the testis is restricted to the haploid germ cells in the seminiferous epithelium. However, it is not present at the ectoplasmic specializations in germ cells but exclusively in the acrosome of the spermatid.

The absence of vezatin in Sertoli cells and the absence of colocalization with markers of the ectoplasmic specialization indicate that vezatin is missing from the adherens junctions of the seminiferous epithelium. In early mouse development, vezatin is essential to the assembly/stability of the E-cadherin-catenins complex at the adherens junction (Hyenne et al. 2005, unpublished results). To our knowledge, Sertoli cells are the only known epithelial cells lacking vezatin at cell-cell 

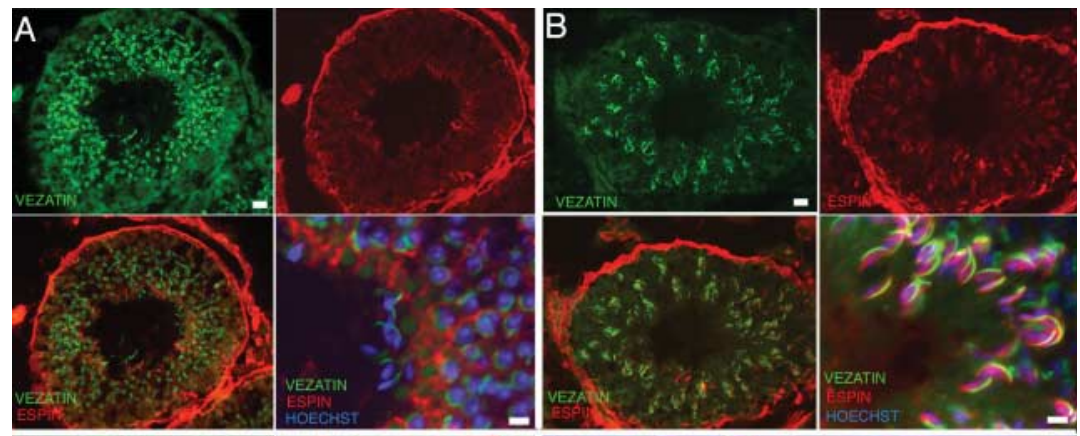

Figure 4 Indirect immunofluorescence localization of vezatin in wild-type testis cryosections, observed with a conventional immunofluorescence microscope. (A and B) A wild-type tubule at stage 7 $(\mathrm{S} 7+\mathrm{S} 16)$ and at stage 9 (S9; see legend in Fig. 2) respectively, stained for vezatin (green), nuclei (blue), and espin (red) and superposition of all three stainings. Faint staining in the basal compartment in $A$ is a non-specific reaction in this labeling
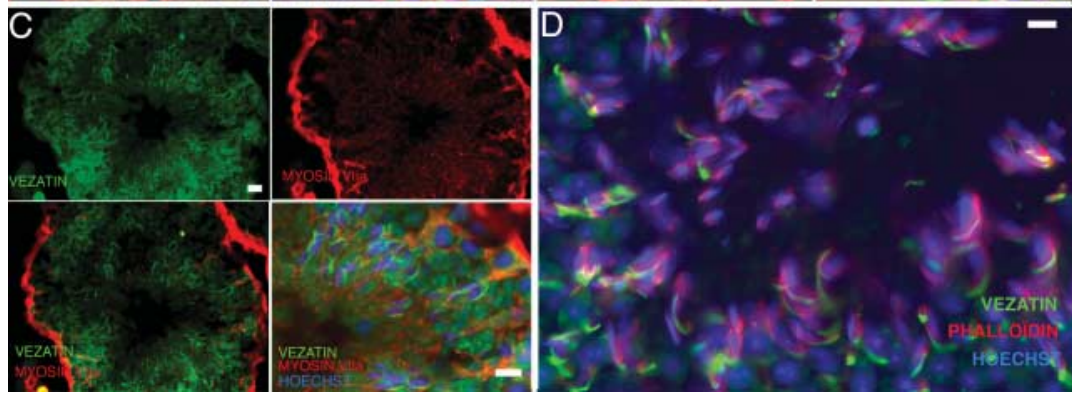

experiment. (C) A wild-type tubule at stage 10 (S10) stained for vezatin (green), nuclei (blue), and myosin VIla and superposition of all three stainings. (D) A wild-type tubule at stage 12 (S12) simultaneously stained for vezatin (green), nuclei (blue), and phalloidin (red) at higher magnification. Phalloidin and vezatin labeling both follow the edges of the spermatid heads but do not colocalize. Bars: (A-C) 30 and $10 \mu \mathrm{m}$ at higher magnification respectively. (D) $5 \mu \mathrm{m}$.

junctions so far. This finding reinforces their unique character, with a molecular composition different from that of a conventional epithelium. In the seminiferous epithelium, the basal Sertoli-Sertoli cells junctions and the apical Sertoli-germ cells junctions both contain the classical cadherin-catenin complex as well as other cytoskeleton-associated proteins including myosin VIla (Hasson et al. 1997, Wolfrum et al. 1998, Lee et al. 2003). How myosin VIla, exclusively expressed in Sertoli cells, is targeted to the apical ectoplasmic specialization in the absence of one of its transmembrane partner, vezatin, remains to be elucidated. Recent studies indicate that myosin VIla is integrated together with other actin-binding proteins into complex protein networks (Boeda et al. 2002, Adato et al. 2005, Reiners et al. 2005, 2006, Senften et al. 2006). In the testis, members of these complexes may target myosin VIla to the spermatid membrane.

During spermatid differentiation, vezatin expression follows acrosomal development. The biogenesis of the acrosome may be divided into four main phases: the Golgi phase (steps S1-S3), the cap phase (steps S4-S7), the acrosome phase (steps S8-S12), and the maturation phase (steps S13-S16) (Abou-Haila \& Tulsiani 2000). PNA/WGA lectins stained all stages of acrosome development. An overlay of the lectins and of the antivezatin antibody labeling shows extensive areas of overlapping staining indicating colocalization of vezatin with glycoproteins of the acrosomal regions. At first, during the early step of the Golgi phase, no localization with the proacrosomal Golgi-derived granule (granulosome) is observed in round spermatids (step S1). However, soon after, vezatin localized around the granulosome in a ring encircling a central hole corresponding to the size of the granulosome and devoid of vezatin staining (steps S2-S3 of the Golgi phase). During the cap and acrosomal phases, as spermatid metamorphoses into an elongated spermatid, vezatin staining is enlarging over the nucleus following the acrosome flattening and its sac-like structure shaping. During the acrosomal phase, vezatin staining overlaps the PNA/WGA labeling which demarcates the sickleshaped acrosome. At the maturation phase, vezatin is found as a rim along the anterior aspect of the spermatid, partially overlapping with the larger lectin staining and thus forming a cap-like structure at the periphery of the acrosome. This sublocalization is even more evident in mature sperm. At the ultrastructural level, immunogold staining was compatible with a progressive appearance of the protein to the acrosomal compartment in spermatids.

The acrosome is considered analogous to a large secretory granule. However, its biogenesis is complex and remains debated (Abou-Haila \& Tulsiani 2000, Ramalho-Santos et al. 2001, 2002). Its continuous maturation involves membrane docking and fusion and the participation of the Golgi apparatus. In the Golgi phase, numerous small proacrosomal vesicles coalesce into a single spherical vesicle that attaches to the nucleus. At that stage, acrosomal specific proteins are usually transported from the endoplasmic reticulum to the developing acrosome, through the Golgi apparatus. However, other acrosomal proteins, like acrin 2, may be transported to the acrosome without transport through the Golgi (Toshimori 1998, Yoshinaga et al. 2001). During the cap phase, the acrosomal vesicle starts to grow as a result of the progressive fusion of Golgiderived membranes and possibly of membranes derived from the lysozome-endosome system (West \& Willison 1996). The acrosomal vesicle flattens and spreads over 

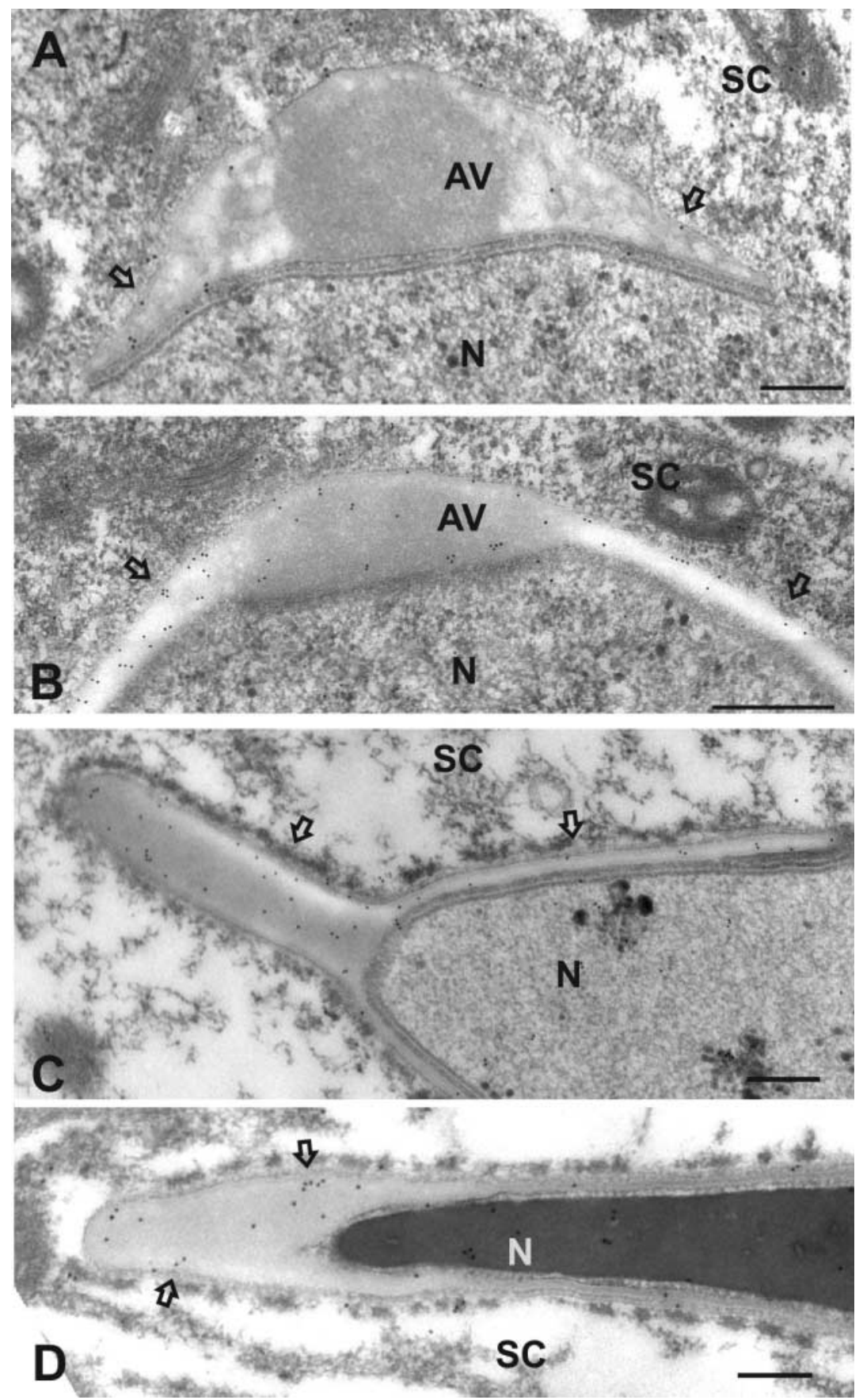

Figure 5 Immunoelectron microscopy analyses of ultrathin sections through mouse wild-type germ cells immunogold labeled with affinity purified antibodies against the intracellular domain of vezatin and $10 \mathrm{~nm}$ colloidal gold coupled antirabbit Fab fragments as secondary antibody. Sertoli cells (SC) were not stained with the anti-vezatin antibody. In germ cells, the localization of vezatin is almost restricted to the acrosome of spermatids. Spermatid in testicular tissue: (A) Golgi phase, sparse immunogold labeling of vezatin is restricted to the growing acrosome (arrows). In contrast, the acrosomal granule (referred here as 'acrosomal vesicle', AV) lacks vezatin labeling. (B) Cap phase, prominent vezatin labeling is present in the acrosome (arrows); less staining appears in the reduced AV. (C) Elongation or acrosomal phase, prominent vezatin labeling is present in the acrosome (arrows). (D) Maturation phase, vezatin is predominantly localized in the acrosome (arrows). Note: some antibodies bind to the condensed DNA of the sperm nucleus $(\mathrm{N})$ in $\mathrm{D}$ but not to the less dense nuclear DNA in a-c. Bars: $500 \mathrm{~nm}$. the nucleus, covering two-thirds of its surface. Later, the Golgi apparatus, which has migrated at the opposite pole of the developing acrosome is discarded in the cytoplasmic droplet (Ramalho-Santos et al. 2002).

By contrast to several acrosomal specific proteins, which are already expressed in the Golgi apparatus of diploid germ cells (Kremling et al. 1991, RamalhoSantos et al. 2002), we did not find any vezatin labeling in secondary spermatocytes. Moreover, it is not found within the spherical proacrosomal electron-dense
Golgi-derived granule when it first migrated to the nuclear envelope in the early round spermatid (step S1). Vezatin first appears when the acrosome expands (step S2), then it follows the morphological changes of the acrosome throughout spermatid differentiation, including in sperm.

Our ultrastructural data, i.e., from the epididymes of mouse and three other mammals (pig, bovine, and human sperm), suggest that vezatin also resides at the outer acrosomal membrane in mammalian sperms. 

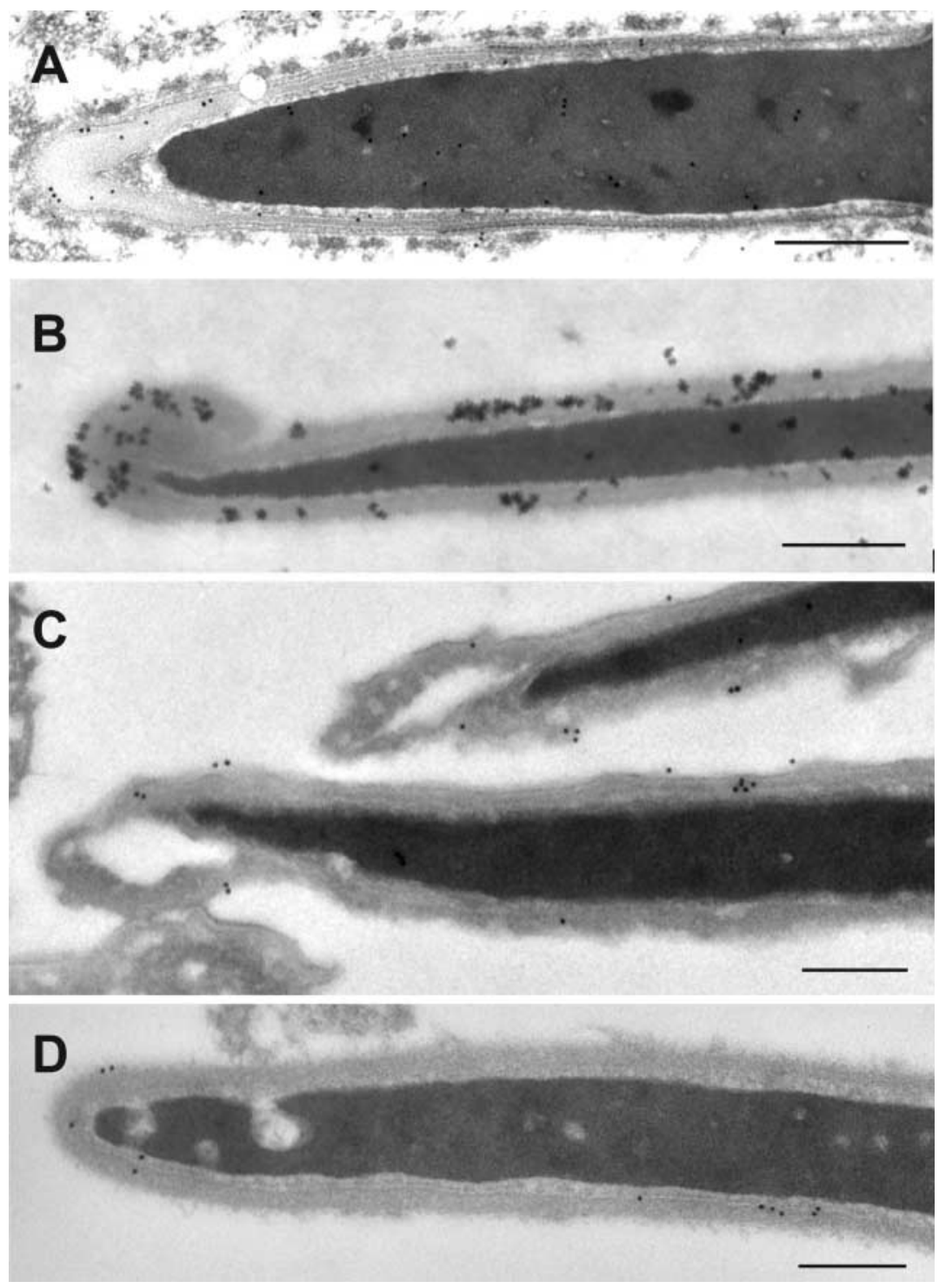

Figure 6 Electron micrographs of sections through isolated sperm of diverse mammalian species illustrating subcellular localization of vezatin: (A) mouse sperm; (B) pig sperm; (C) bovine sperm; and (D) human sperm. In ultrathin sections through sperms of all mammalian species analyzed, vezatin labeling is predominantly localized at the outer acrosomal membrane and the small cytoplasmic portion of the sperm head as well as the region of the acrosome. In contrast, only a few colloidal particles are found to bind to the condensed DNA in the nucleus (N) (indirect immunogold labeling: a, c, and $\mathrm{d}$ : $10 \mathrm{~nm}$ colloidal gold coupled anti-rabbit Fab fragment; $1.4 \mathrm{~nm}$ Nanogold conjugated to antirabbit Fab fragments. (b) Bars: $500 \mathrm{~nm}$.
Acrosomal membranes are distinguishable into two functional domains, the inner acrosomal membrane facing the nucleus and the outer acrosomal membrane attached to the plasma membrane (Ramalho-Santos et al. 2002, Toshimori \& Ito 2003). They are defined when the acrosome-nucleus complex rotates at the end of the cap phase so that the acrosome faces the basal membrane (Russell et al. 1990). This membrane compartmentalization is of major importance for the acrosomal reaction at fertilization, when the outer acrosomal membrane fuses with the plasma membrane in capacitated sperm. The molecular changes involved in the priming of the outer acrosomal membrane and the plasma membrane prior to fusion during the acrosomal reaction are, however, not fully understood. How exactly vezatin, an integral membrane protein, is translocated at the outer acrosomal membrane remains to be investigated. Although proteins belonging to the membrane trafficking machinery have been described throughout spermiogenesis and during epididymal transit (Peterson et al. 1992, Ramalho-Santos et al. 2001), the mechanisms for vectorial translocation during the gradual formation and differentiation of the acrosome or 'acrosomogenesis', are still under investigation (Kierszenbaum 2000).

In conclusion, we report here a novel localization for vezatin, a ubiquitous transmembrane protein associated with adherens junction in most epithelial cells. Vezatin is exclusively expressed in the acrosome of the male germ cells, in the mouse. Vezatin is progressively concentrated as a thin and peripheral marker over the anterior half of the forming acrosomal system in spermatids and localizes at the outer acrosomal membrane in more mature sperms. This observation suggests that during the final step of spermiogenesis or during germ maturation and remodeling in the epididymis, vezatin could play a role independent of its known function at adherens junctions of conventional epithelial cells. 


\section{Acknowledgements}

This work was supported by research grants R0375/38 and R0475/100 from the Ligue Contre le Cancer (BM), the DFG (UW), and the FAUN-Stiftung, Nürnberg (UW). Vincent Hyenne was the recipient of fellowships from the Ministère de l'Education et de la Recherche Technologique (MENRT) and from the Fondation pour la Recherche Médicale (FRM). We are grateful to Dr Günther Kamp (Institut für Zoologie, Johannes Gutenberg-Universität, Germany) for kindly providing sperm specimens for immunoelectron microscopic analyses, to Drs Elisabeth Verpy and Michel Leibovici (INSERMUMRS587/Institut Pasteur/Collège de France, France) for generous gift of frozen samples, and to Dr Anne-Marie Courtot (INSERMU566/CEA) for support. We thank Gabi Stern-Schneider and Elizabeth Sehn (Institut für Zoologie, Johannes GutenbergUniversität) for excellent technical help. The authors declare that there is no conflict of interest that would prejudice the impartiality of this scientific work.

\section{References}

Abou-Haila A \& Tulsiani DR 2000 Mammalian sperm acrosome: formation, contents, and function. Archives of Biochemistry and Biophysics 379 173-182.

Adato A, Michel V, Kikkawa Y, Reiners J, Alagramam KN, Weil D, Yonekawa H, Wolfrum U, El-Amraoui A \& Petit C 2005 Interactions in the network of Usher syndrome type 1 proteins. Human Molecular Genetics 14 347-356.

Bartles JR, Wierda A \& Zheng L 1996 Identification and characterization of espin, an actin-binding protein localized to the F-actinrich junctional plaques of Sertoli cell ectoplasmic specializations. Journal of Cell Science 109 1229-1239.

Benoff S 1997 Carbohydrates and fertilization: an overview. Molecular Human Reproduction 3 599-637.

Boeda B, El-Amraoui A, Bahloul A, Goodyear R, Daviet L, Blanchard S, Perfettini I, Fath KR, Shorte S, Reiners J et al. 2002 Myosin VIla, harmonin and cadherin 23, three Usher I gene products that cooperate to shape the sensory hair cell bundle. EMBO Journal 21 6689-6699.

Bouchard MJ, Dong Y, McDermott BM Jr, Lam DH, Brown KR, Shelanski M, Bellve AR \& Racaniello VR 2000 Defects in nuclear and cytoskeletal morphology and mitochondrial localization in spermatozoa of mice lacking nectin-2, a component of cell-cell adherens junctions. Molecular and Cellular Biology 20 2865-2873.

Burkin H, Zhao L \& Miller D 2004 CASK is in the mammalian sperm head and is processed during epididymal maturation. Molecular Reproduction and Development 68 500-506.

Byers SW, Sujarit S, Jegou B, Butz S, Hoschutzky H, Herrenknecht K, MacCalman C \& Blaschuk OW 1994 Cadherins and cadherinassociated molecules in the developing and maturing rat testis. Endocrinology 134 630-639.

Cheng CY \& Mruk DD 2002 Cell junction dynamics in the testis: sertoli-germ cell interactions and male contraceptive development Physiological Reviews 82 825-874.

Christova Y, James P, Mackie A, Cooper TG \& Jones R 2004 Molecular diffusion in sperm plasma membranes during epididymal maturation. Molecular and Cellular Endocrinology 216 41-46.

Danscher G 1981 Light and electron microscopic localization of silver in biological tissue. Histochemistry 71 177-186.

DeBellefeuille S, Hermo L, Gregory M, Dufresne J \& Cyr DG 2003 Catenins in the rat epididymis: their expression and regulation in adulthood and during postnatal development. Endocrinology 144 5040-5049.
Drenckhahn D \& Groschel-Stewart U 1980 Localization of myosin, actin, and tropomyosin in rat intestinal epithelium: immunohistochemical studies at the light and electron microscope levels. Journal of Cell Biology 86 475-482.

Fujita E, Kouroku Y, Ozeki S, Tanabe Y, Toyama Y, Maekawa M, Kojima N, Senoo H, Toshimori K \& Momoi T 2006 Oligo-asthenoteratozoospermia in mice lacking RA175/TSLC1/SynCAM/IGSF4A, a cell adhesion molecule in the immunoglobulin superfamily. Molecular and Cellular Biology 26 718-726.

Gliki G, Ebnet K, Aurrand-Lions M, Imhof BA \& Adams RH 2004 Spermatid differentiation requires the assembly of a cell polarity complex downstream of junctional adhesion molecule-C. Nature 431 320-324.

Goossens S \& van Roy F 2005 Cadherin-mediated cell-cell adhesion in the testis. Frontiers in Bioscience 10 398-419.

Hasson T, Walsh J, Cable J, Mooseker MS, Brown SD \& Steel KP 1997 Effects of shaker-1 mutations on myosin-VIla protein and mRNA expression. Cell Motility and the Cytoskeleton 37 127-138.

Hyenne V, Louvet-Vallée S, El-Amraoui A, Petit C, Maro B \& Simmler M-C 2005 Vezatin, a protein associated to adherens junctions, is required for mouse blastocyst morphogenesis. Developmental Biology 287 180-191.

Inagaki $M$, Irie $K$, Ishizaki $H$, Tanaka-Okamoto $M$, Morimoto $K$, Inoue E, Ohtsuka T, Miyoshi J \& Takai Y 2005 Roles of cell-adhesion molecules nectin 1 and nectin 3 in ciliary body development. Development 132 1525-1537.

Johnston D, Jelinsky S, Bang H, Dicandalero P, Wilson E, Kopf G \& Turner T 2005 The murine epididymal transcriptome: transcriptional profiling of segmental gene expression in the epididymis. Biology of Reproduction 73 404-413.

Kierszenbaum AL 2000 Fusion of membranes during the acrosome reaction: a tale of two SNAREs. Molecular Reproduction and Development 57 309-310.

Kotaja N, Kimmins S, Brancorsini S, Hentsch D, Vonesh J, Davidson I, Parvinen M \& Sassone-Corsi P 2004 Preparation, isolation and characterization of stage-specific spermatogenic cells for cellular and molecular analysis. Nature Methods $1249-254$.

Kremling H, Keime S, Wilhelm K, Adham IM, Hameister H \& Engel W 1991 Mouse proacrosin gene: nucleotide sequence, diploid expression, and chromosomal localization. Genomics 11 828-834.

Kussel-Andermann P, El-Amraoui A, Safieddine S, Nouaille S, Perfettini I, Lecuit M, Cossart P, Wolfrum U \& Petit C 2000 Vezatin, a novel transmembrane protein, bridges myosin VIIA to the cadherin-catenins complex. EMBO Journal 19 6020-6029.

Lee NP, Mruk D, Lee WM \& Cheng CY 2003 Is the cadherin/catenin complex a functional unit of cell-cell actin-based adherens junctions in the rat testis? Biology of Reproduction 68 489-508.

Lessard J 1988 Two monoclonal antibodies to actin: one muscle selective and one generally reactive. Cell Motility and the Cytoskeleton 10 349-362.

Mruk DD \& Cheng CY 2004 Sertoli-Sertoli and Sertoli-germ cell interactions and their significance in germ cell movement in the seminiferous epithelium during spermatogenesis. Endocrine Reviews 25 747-806.

Mulholland DJ, Dedhar S \& Vogl AW 2001 Rat seminiferous epithelium contains a unique junction (ectoplasmic specialization) with signaling properties both of cell/cell and cell/matrix junctions. Biology of Reproduction 64 396-407.

Newton SC, Blaschuk OW \& Millette CF $1993 \mathrm{~N}$-cadherin mediates Sertoli cell-spermatogenic cell adhesion. Developmental Dynamics $1971-13$.

Orgebin-Crist M 1967 Sperm maturation in rabbit epididymis. Nature 216 816-818.

Ozaki-Kuroda K, Nakanishi H, Ohta H, Tanaka H, Kurihara H, Mueller S, Irie K, Ikeda W, Sakai T, Wimmer E et al. 2002 Nectin couples cell-cell adhesion and the actin scaffold at heterotypic testicular junctions. Current Biology 12 1145-1150. 
Palombi F, Salanova M, Tarone G, Farini D \& Stefanini M 1992 Distribution of beta 1 integrin subunit in rat seminiferous epithelium. Biology of Reproduction 47 1173-1182.

Peterson RN, Bozzola J \& Polakoski K 1992 Protein transport and organization of the developing mammalian sperm acrosome. Tissue Cell 24 1-15.

Ramalho-Santos J, Moreno RD, Wessel GM, Chan EK \& Schatten G 2001 Membrane trafficking machinery components associated with the mammalian acrosome during spermiogenesis. Experimental Cell Research 267 45-60.

Ramalho-Santos J, Schatten G \& Moreno RD 2002 Control of membrane fusion during spermiogenesis and the acrosome reaction. Biology of Reproduction 67 1043-1051.

Reiners J, van Wijk E, Marker T, Zimmermann U, Jurgens $K$, Te Brinke H, Overlack N, Roepman R, Knipper M, Kremer $\mathrm{H}$ et al. 2005 The scaffold protein harmonin (USH1C) provides molecular links between Usher syndrome type 1 and type 2. Human Molecular Genetics 14 3933-3943.

Reiners J, Nagel-Wolfrum K, Jurgens K, Marker T \& Wolfrum U 2006 Molecular basis of human Usher syndrome: deciphering the meshes of the Usher protein network provides insights into the pathomechanisms of the Usher disease. Experimental Eye Research 83 97-119.

Russell L, Ettlin R, Sinha Hikim A \& Clegg E 1990 Mammalian Spermatogenesis, St Louis: Cache River Press.

Salanova M, Stefanini M, De Curtis I \& Palombi F 1995 Integrin receptor alpha 6 beta 1 is localized at specific sites of cell-to-cell contact in rat seminiferous epithelium. Biology of Reproduction $\mathbf{5 2}$ 79-87.

Senften M, Schwander M, Kazmierczak P, Lillo C, Shin JB, Hasson T, Geleoc GS, Gillespie PG, Williams D, Holt JR et al. 2006 Physical and functional interaction between protocadherin 15 and myosin VIla in mechanosensory hair cells. Journal of Neuroscience $\mathbf{2 6}$ 2060-2071.
Sullivan R, Saez F, Girouard J \& Frenette G 2005 Role of exosomes in sperm maturation during the transit along the male reproductive tract. Blood Cells, Molecules and Diseases 35 1-10.

Toshimori K 1998 Maturation of mammalian spermatozoa: modifications of the acrosome and plasma membrane leading to fertilization. Cell and Tissue Research 293 177-187.

Toshimori K \& Ito C 2003 Formation and organization of the mammalian sperm head. Archives of Histology and Cytology 66 383-396.

West AP \& Willison KR 1996 Brefeldin A and mannose 6-phosphate regulation of acrosomic related vesicular trafficking. European Journal of Cell Biology 70 315-321.

Wolfrum U 1992 Cytoskeletal elements in arthropod sensilla and mammalian photoreceptors. Biologie Cellulaire 76 373-381.

Wolfrum U \& Salisbury JL 1998 Expression of centrin isoforms in the mammalian retina. Experimental Cell Research 242 10-17.

Wolfrum U, Liu X, Schmitt A, Udovichenko IP \& Williams DS 1998 Myosin VIla as a common component of cilia and microvilli. Cell Motility and the Cytoskeleton 40 261-271.

Yoshinaga K \& Toshimori K 2003 Organization and modifications of sperm acrosomal molecules during spermatogenesis and epididymal maturation. Microscopy Research and Technique 61 39-45.

Yoshinaga K, Tanii I, Oh-oka T \& Toshimori K 2001 Changes in distribution and molecular weight of the acrosomal protein acrin2 (MC41) during guinea pig spermiogenesis and epididymal maturation. Cell and Tissue Research 303 253-261.

Zhou C, Zhang Y, Xiao L, Zheng M, Leung K, Chan M, Lo P, Tsang L, Wong H, Ho L et al. 2004 An epididymis-specific beta-defensin is important for the initiation of sperm maturation. Nature Cell Biology 6 458-464.

Received 13 October 2006

First decision 9 November 2006

Accepted 11 December 2006 